\title{
PÓS-ALTA DE HANSENÍASE: PREVALÊNCIA DE INCAPACIDADES FíSICAS E SOBREPOSIÇÃO DE DOENÇAS
}

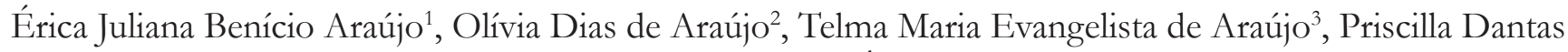 \\ Almeida ${ }^{4}$, Inara Viviane de Oliveira Sena ${ }^{5}$, Érica de Alencar Rodrigues Neri ${ }^{5}$
}

\begin{abstract}
RESUMO: Objetivo: caracterizar a prevalência de incapacidades físicas em indivíduos acometidos por hanseníase no pós-alta e sua relação com as doenças crônicas. Método: a população da pesquisa foi de 603 casos novos de hanseníase notificados entre 2001 e 2014 em municípios endêmicos no Piauí. Os dados foram coletados mediante aplicação de questionários e avaliação neurológica simplificada. Análises estatísticas foram realizadas por distribuição de frequência, testes Qui-quadrado de Pearson e Exato de Fisher. Resultados: observouse a prevalência de incapacidade física de $70,43 \%$, e associação significativa com a forma clínica multibacilar, sexo masculino, idade avançada e baixa escolaridade $(p<0,01)$. Conclusão: a sobreposição de doenças não se comportou como um fator de risco, mas constatou-se frequência importante de pessoas. Registra-se a necessidade de planejamento de ações de saúde mais adequadas à população, fortalecendo a longitudinalidade do cuidado frente às condições crônicas.

DESCRITORES: Hanseníase; Pessoas com deficiência; Epidemiologia; Doença crônica; Saúde pública.
\end{abstract}

\section{POST-DISCHARGE OF SUBJECTS WITH LEPROSY: PREVALENCE OF PHYSICAL DISABILITIES AND OVERLAPPING OF OTHER DISEASES}

\begin{abstract}
Objective: To characterize the prevalence of physical disabilities in individuals affected by post-discharge leprosy and its relationship with chronic diseases.Method: The study population consisted of 603 new cases of leprosy reported between 2001 and 2014 in endemic municipalities in the state of Piauí. Data was collected through questionnaires and simplified neurological evaluation. Statistical analyzes were performed by frequency distribution, Pearson's Chi-square test and Fisher's Exact test. Results. The prevalence of physical disability was $70.43 \%$, and there was a significant association with the multibacillary clinical form, male gender, old age and low educational level $(p<0.01)$.Conclusion:Overlapping of diseases was not a risk factor for the development of physical disabilities, but there was a higher frequency of diabetic and hypertensive subjects in the group with physical disabilities. It is necessary to plan health actions that are more suitable to the population, strengthening the longitudinality of care to chronic conditions.

DESCRIPTORS: Leprosy; Disabled people; Epidemiology; Chronic disease; Public health.
\end{abstract}

\section{POST-ALTA DE LEPRA: PREVALENCIA DE DISCAPACIDADES FÍSICAS Y SUPERPOSICIÓN DE ENFERMEDADES}

RESUMEN: Objetivo: Caracterizar la prevalencia de discapacidades físicas en individuos afectados por lepra luego del alta y su relación con las enfermedades crónicas. Método: La población del estudio fue de 603 casos nuevos de lepra notificados entre 2001 y 2014 en municipios endémicos de Piauí. Datos recolectados mediante aplicación de cuestionarios y evaluación neurológica simplificada. Se realizaron análisis estadísticos de distribución de frecuencia, tests de Chi-cuadrado de Pearson y Exacto de Fisher. Resultados: Se observó prevalencia de discapacidad física del 70,43\%, y asociación significativa con la forma clínica multibacilar, sexo masculino, edad avanzada y baja escolarización $(p<0,01)$. Conclusión: La superposición de enfermedades no constituyó un factor de riesgo, aunque se la constató en importante cantidad de personas. Se registra la necesidad de planificación de acciones de salud más adecuadas a la población, fortaleciendo la atención longitudinal frente a las condiciones crónicas.

DESCRIPTORES: Lepra; Personas con Discapacidad; Epidemiología; Enfermedad Crónica; Salud Pública.

${ }^{1}$ Enfermeira. Universidade Federal do Piauí. Teresina, PI, Brasil.

${ }^{2}$ Enfermeira. Doutora em Enfermagem. Docente de Enfermagem da Universidade Federal do Piauí. Teresina, PI, Brasil.

${ }^{3}$ Enfermeira. Doutora em Enfermagem em Saúde Coletiva. Docente de Enfermagem da Universidade Federal do Piauí. Teresina, PI, Brasil.

${ }^{4}$ Enfermeira. Mestre em Saúde e Comunidade. Universidade Federal do Piauí. Teresina, PI, Brasil.

${ }^{5}$ Enfermeira. Mestre em Enfermagem. Universidade Federal do Piauí. Teresina, PI, Brasil. 


\section{- INTRODUÇÃO}

A hanseníase atualmente ainda se configura como um problema de saúde pública no Brasil, tanto pela amplitude das consequências físicas e socioeconômicas, quanto pela endemicidade em que se encontram alguns estados brasileiros ${ }^{(1)}$.

A doença se manifesta por alterações dermatoneurológicas, como lesões cutâneas e em nervos periféricos, acometendo principalmente olhos, mãos, e pés, gerando alterações motoras e sensoriais com impactos significativos do ponto de vista físico, social e econômico(2).

Segundo a Organização Mundial de Saúde (OMS), em 2016, foram registrados em 14 países endêmicos, 143 países reportaram 214.783 casos novos de hanseníase, o que representa uma taxa de detecção de 2,9 casos por 100 mil habitantes. O Brasil aparece classificado como de alta carga para a doença, sendo o segundo com o maior número de casos novos registrados no mundo ${ }^{(3)}$.

Apesar da redução significativa dos casos novos de hanseníase nos últimos 10 anos, a detecção se mantém alta no Brasil, com distribuição não uniforme de casos e concentração em áreas hiperendêmicas, principalmente nas regiões Norte, Centro-oeste e Nordeste. Em 2014, foi registrado um total de 31.064 de casos novos no Brasil, apresentando taxa de detecção geral de 15,32 por 100 mil habitantes. No Piauí, apesar da importante redução no coeficiente de prevalência da hanseníase, que no ano de 2014 apresentou 2,49 casos/10 mil habitantes, o estado ainda demanda intensificação das ações para eliminação da doença ${ }^{(4)}$.

Acompanha essa realidade a proporção de pacientes detectados com incapacidades físicas instaladas, evidência de retardo no diagnóstico e comprometimento neural. Cerca de $20 \%$ das pessoas atingidas pela hanseníase podem sofrer incapacidades ou problemas psicossociais, com necessidade de algum tipo de apoio e/ou reabilitação. No Brasil, 6,6\% entre os casos novos avaliados no diagnóstico apresentaram grau 2 de incapacidade, no Piauí a proporção é de 5,7\%. Logo, além do tratamento quimioterápico, faz-se necessário ressaltar a importância das técnicas de prevenção, de controle e de tratamento das incapacidades e deformidades, como atenção integral à pessoa com hanseníase ${ }^{(3,5-6)}$.

No contexto de transições da saúde pública, a hanseníase representa condição de saúde crônica, que acomete crianças e adultos, com elevado potencial de estar associada a outras condições crônicas e degenerativas, potencializando-as ou tendo a sua história natural modificada. Com isto, amplia, em populações vulneráveis e negligenciadas, não apenas a sua carga específica, mas também a de outras condições, trazendo grandes desafios aos sistemas de saúde dos países endêmicos. O seu contexto social e fato da hanseníase ainda ser considerada negligenciada faz com que as pessoas acometidas no momento do pós-alta não tenham um acompanhamento contínuo ${ }^{(7)}$.

O comportamento da hanseníase quando relacionada a outras doenças crônicas coexistentes como a diabetes e a hipertensão arterial ainda é pouco conhecido. No entanto, percebe-se a relevância dessa relação hanseníase e doenças crônicas no que diz respeito à investigação do possível potencial incapacitante, característico da hanseníase, associado a essas doenças.

Tendo em vista a situação da hanseníase como problema de saúde pública no Brasil e no Estado do Piauí, a variedade de aspectos que podem estar envolvidos com o desenvolvimento de incapacidades físicas e a necessidade de conhecer se há relação ou não do agravamento desta situação quando associada a outras doenças crônicas, o presente estudo teve como objetivo caracterizar a prevalência de incapacidades físicas em indivíduos acometidos por hanseníase no pós-alta e sua relação com a sobreposição de doenças crônicas.

\section{- MÉTODO}

Este estudo faz parte de um macroprojeto da Pós-Graduação em Enfermagem da Universidade Federal do Piauí (UFPI), denominado "INTEGRAHANS PIAUÍ: abordagem integrada dos aspectos clínicos, epidemiológicos, operacionais e psicossociais da Hanseníase em municípios de alta endemicidade do estado do Piauí". 
Trata-se de um estudo transversal realizado em dois municípios de alta endemicidade do estado do Piauí: Floriano (clustser 1), considerado hiperendêmico para Hanseníase, com uma população de 58.803 mil pessoas e área territorial de $3.409,649$ km², e Picos (cluster 6), município endêmico, com população de 73.414 mil habitantes e área territorial de 577.304 km².

A população de estudo foi composta a partir da consolidação dos dados do Sistema de Informação de Agravos de Notificação (SINAN) referente aos casos novos que foram notificados em Floriano e Picos no período de 2001 a 2014, totalizando 1765 casos.

Do total de casos, 751 (42,45\%) não foram localizados. Dos 1.014 (57,45\%) casos localizados, 321 $(31,66 \%)$ não foram abordados por motivo de mudança de endereço, óbito ou por não terem sido encontrados no momento da abordagem domiciliar. Um total de 90 (12,99\%) pessoas da população que foi abordada não consentiu em fazer parte da pesquisa.

Foram avaliados no estudo, portanto, 603 pessoas, sendo 311 (51,58\%) de Floriano e 292 (48,42\%) de Picos. Definiu-se como critério de inclusão que os participantes deveriam ter sido diagnosticados e tratados nos referidos municípios e estarem no momento pós-alta, ou seja, terem recebido alta por cura da Hanseníase. Foram excluídas as pessoas que migraram para outro município.

A coleta de dados ocorreu no período de julho de 2015 a junho de 2016. Realizou-se o mapeamento dos casos e confirmação dos endereços confrontando os dados do SINAN com os endereços constantes no Sistema de Cadastramento de Usuários do Sistema Único de Saúde (CADSUS) e os óbitos, com o Sistema de Informação de Mortalidade (SIM). Após a localização dos domicílios, prosseguiu-se para a realização das visitas aos casos de hanseníase pela equipe de abordagem domiciliar, convidando-os a comparecerem em locais e data previamente estabelecidas para participarem da pesquisa, sendo esclarecidos todos os objetivos e procedimentos.

Os dados foram coletados mediante entrevista padronizada, com aplicação de questionários estruturados e realização de exame clínico aos pacientes pelos profissionais envolvidos no projeto e colaboradores, previamente capacitados. Os casos que não comparecerem foram revisitados nos seus domicílios, pela equipe de abordagem domiciliar, realizando-se até três retornos ao domicílio, além de telefonemas quando possível, reafirmando o convite.

O maior grau de incapacidade física (GIF) identificado na Avaliação Neurológica Simplificada preconizada pelo Ministério da Saúde permitiu a avaliação da magnitude das incapacidades. O grau de incapacidade foi determinado a partir da avaliação neurológica simplificada dos olhos, mãos e pés e o seu resultado expresso em valores que variam de 0 (zero) a 2 (dois): 0 - não há presença de comprometimento neural; 1 - diminuição ou da perda da sensibilidade; 2 - presença de incapacidades e deformidades visíveis ${ }^{(6,8)}$.

Foram investigadas as variáveis socioeconômicas e clínicas como sexo, idade, escolaridade, ocupação, situação conjugal, classificação operacional, forma clínica, escore olhos, mãos e pés (OMP), ocorrência de reações hansênicas, dados do perfil clínico geral como a presença de doenças crônicas (diabetes e hipertensão) e outras comorbidades, e dados relacionados ao serviço, como a frequência da visita de Agente Comunitário de Saúde (ACS) durante e após o tratamento com Poliquimioterapia (PQT) e a avaliação neurológica simplificada.

Os dados foram digitados e armazenados no programa Epi-Info versão 7.1.5.0 e analisados no programa Stata 13.0. Para análise, além da abordagem descritiva, foi utilizado o teste Qui-quadrado de Pearson e o teste Exato de Fisher, com nível de confiança de 95\% e nível de significância de 5\%.

O trabalho foi aprovado pelo Comitê de Ética em Pesquisa da UFPI, sob Parecer $\mathrm{n}^{\mathrm{o}}$ 1.115.818.

\section{- RESULTADOS}

A prevalência do Grau de Incapacidade Física (GIF) foi identificada nos casos avaliados (70,43\%, $\mathrm{n}=424)$, entre GIF $1(54,32 \%, \mathrm{n}=327)$ e GIF $2(16,11 \% \mathrm{n}=97)$.

A maioria da amostra estudada pertenceu ao sexo feminino $(52,07 \%, n=314)$, apresentava a média de 
idade de 51,6 anos, variando de 10 a 102 anos, com predominância de idosos (36,49\%, n=216), casados ou unidos $(50,68 \%, n=300)$, tinha o ensino fundamental incompleto $(46,06 \%, n=269)$ e autodeclararam pardos ou negros $(72,48 \%, \mathrm{n}=424)$.

Houve uma maior frequência de casos paucibacilares $(53,57 \%, n=323)$ e como forma clínica predominante observou-se a indeterminada $(36,36 \%, \mathrm{n}=216)$, seguida pela boderline $(28,62 \%, \mathrm{n}=170)$. Quanto à ocorrência de reações hansênicas 22,18\% (n=122) tiveram algum episódio reacional.

Constatou-se maior frequência de incapacidade física em pessoas do sexo masculino, com idade superior a 60 anos, com tendência de ocorrência de incapacidade em relação ao aumento da idade $(p<0,01)$. A idade média das pessoas com incapacidade física foi de 55,11 anos. Observou-se que as pessoas em situação conjugal separado, divorciado ou viúvo sofreram mais incapacidades $(p<0,01)$, assim como em pessoas com nenhuma escolaridade $(p<0,01)$. A associação com a cor da pele autorreferida não teve significância estatística (Tabela 1).

Tabela 1 - Caracterização clínica e sociodemográfica dos casos de Hanseníase segundo a presença de Incapacidade Física. Floriano e Picos, Pl, 2017 (continua)

\begin{tabular}{|c|c|c|c|c|c|c|}
\hline \multirow[t]{2}{*}{ Variáveis } & \multicolumn{2}{|c|}{ Sem inc. física } & \multicolumn{2}{|c|}{ Com inc. física } & \multirow{2}{*}{$\begin{array}{c}\text { TOTAL } \\
\mathbf{N}\end{array}$} & \multirow{2}{*}{ p valor } \\
\hline & $\mathbf{N}$ & $\%$ & $\mathbf{n}$ & $\%$ & & \\
\hline \multicolumn{7}{|l|}{ Class. operacional $^{\mathrm{a}}$} \\
\hline Paucibacilar & 128 & 39,63 & 195 & 60,37 & 323 & \multirow{2}{*}{0} \\
\hline Multibacilar & 50 & 17,92 & 229 & 82,08 & 279 & \\
\hline \multicolumn{7}{|l|}{ Forma clínicab $^{b}$} \\
\hline Indeterminada & 117 & 92,13 & 10 & 7,87 & 127 & \multirow{4}{*}{0} \\
\hline Tuberculoide & 55 & 87,3 & 8 & 12,7 & 63 & \\
\hline Borderline & 88 & 75,86 & 28 & 24,14 & 116 & \\
\hline Virchowiana & 25 & 41,67 & 35 & 58,33 & 60 & \\
\hline \multicolumn{7}{|l|}{ Reações hansenicas $^{a(1)}$} \\
\hline Não & 127 & 30,09 & 295 & 69,91 & 422 & \multirow{2}{*}{0,43} \\
\hline Sim & 32 & 26,45 & 89 & 73,55 & 121 & \\
\hline \multicolumn{7}{|l|}{ Sexo $^{a}$} \\
\hline Masculino & 68 & 23,61 & 220 & 76,39 & 288 & \multirow{2}{*}{0} \\
\hline Feminino & 110 & 35,03 & 204 & 64,97 & 314 & \\
\hline \multicolumn{7}{|l|}{ Faixa etáriab $^{b}$} \\
\hline$<15$ anos & 5 & 62,5 & 3 & 37,5 & 8 & \multirow{5}{*}{0} \\
\hline 15 a 29 anos & 40 & 54,05 & 34 & 45,95 & 74 & \\
\hline 30 a 44 anos & 47 & 40,87 & 68 & 59,13 & 115 & \\
\hline 45 a 59 anos & 46 & 27,22 & 123 & 72,78 & 169 & \\
\hline$\geq 60$ anos & 33 & 15,49 & 180 & 84,51 & 213 & \\
\hline \multicolumn{7}{|l|}{ Situação conjugal ${ }^{b}$} \\
\hline Solteiro & 59 & 37,34 & 99 & 62,66 & 158 & \multirow{3}{*}{0} \\
\hline Casado/Unido & 89 & 30,07 & 207 & 69,93 & 296 & \\
\hline Separado/Divorciado/Viúvo & 23 & 18,4 & 102 & 81,6 & 125 & \\
\hline \multicolumn{7}{|l|}{ Escolaridade $^{b}$} \\
\hline Analfabeto & 17 & 14,66 & 99 & 85,34 & 116 & \multirow{6}{*}{0} \\
\hline Ens. Fund. Compl. & 12 & 34,29 & 23 & 65,71 & 35 & \\
\hline Ens. Fund.Inc. & 64 & 24,33 & 199 & 75,67 & 263 & \\
\hline Ens. Méd. Compl. & 45 & 49,45 & 46 & 50,55 & 91 & \\
\hline Ens. Méd. Inc. & 16 & 37,21 & 27 & 62,79 & 43 & \\
\hline Ens. Sup. Compl. & 15 & 65,22 & 8 & 34,78 & 23 & \\
\hline
\end{tabular}




\begin{tabular}{lcccccc}
\hline Cor da pele (autorreferida) $^{\mathrm{b}}$ & \multicolumn{1}{l}{} \\
\hline Branca & 26 & 27,66 & 68 & 72,34 & 94 & \\
\hline Parda/Negra & 122 & 29,61 & 290 & 70,39 & 412 \\
\hline Outra & 21 & 31,82 & 45 & 68,18 & 46 & 0,85
\end{tabular}

(a)Teste Qui-quadrado de Pearson; (b) Teste exato de Fisher; ${ }^{(1)} 60$ pessoas não souberam ou não quiseram responder sobre a ocorrência de reações hansênicas.

A frequência de Incapacidade física foi maior em casos multibacilares e de forma clínica Virchowiana $(p<0,01)$. A média dos escores OMP na ocorrência de Incapacidade física foi de 2,87. Não foi encontrada significância estatística na associação com reações hansênicas, no entanto observa-se que, dentre as pessoas que tiveram episódios reacionais, a maioria $(73,55 \%, \mathrm{n}=121)$ encontrou-se com algum grau de incapacidade física no pós-alta da hanseníase (Tabela 1).

Com relação ao acompanhamento pelo serviço de saúde, $77,74 \%(\mathrm{n}=248)$ das pessoas afirmaram terem recebido visita do ACS durante a PQT e tiveram incapacidade física $(p<0,01)$. Dos casos que não receberam a visita do ACS durante o tratamento e após a alta por cura $64,71 \%(n=143)$ e $65,43 \%(n=159)$, respectivamente, apresentaram incapacidade física $(p<0,01)$. Durante o tratamento, 77,61\% $(n=430)$ foram avaliados por meio da avaliação neurológica simplificada (Tabela 2).

Tabela 2 - Assistência recebida pelos serviços de saúde durante e pós PQT segundo a presença de incapacidade física. Floriano e Picos, Pl, 2017

\begin{tabular}{|c|c|c|c|c|c|c|}
\hline \multirow[t]{2}{*}{ Variáveis } & \multicolumn{2}{|c|}{ Sem inc. física } & \multicolumn{2}{|c|}{ Com inc. física } & \multirow{2}{*}{$\frac{\text { TOTAL }}{\mathbf{N}^{1}}$} & \multirow{2}{*}{ p valor } \\
\hline & $\mathbf{n}$ & $\%$ & $\mathbf{n}$ & $\%$ & & \\
\hline \multicolumn{7}{|c|}{ Visita de ACS durante a PQT ${ }^{\mathrm{a}}$} \\
\hline Não & 0 & 35,29 & 143 & 64,71 & 221 & \multirow{2}{*}{0} \\
\hline Sim & 71 & 22,26 & 248 & 77,74 & 319 & \\
\hline \multicolumn{7}{|c|}{ Visita de ACS no pós-PQTa } \\
\hline Não & 84 & 34,57 & 159 & 65,43 & 243 & \multirow{2}{*}{0} \\
\hline Sim & 74 & 24,26 & 231 & 75,74 & 305 & \\
\hline \multicolumn{7}{|c|}{ Avaliados (ANS) $^{a}$} \\
\hline Não & 31 & 25 & 93 & 75 & 124 & \multirow{2}{*}{0,27} \\
\hline Sim & 129 & 30 & 301 & 70 & 430 & \\
\hline
\end{tabular}

${ }^{(a)}$ Teste Qui-quadrado de Pearson; ${ }^{(b)}$ Teste exato de Fisher; ${ }^{(1)}$ Os números totais (n) diferem em decorrência de valores missing.

As doenças mais identificadas entre todos os participantes do estudo foram diabetes $(12,03 \%, \mathrm{n}=71)$ e hipertensão arterial $(24,45 \%, n=145)$. Foi obtido resultado significativo apenas na associação com a hipertensão arterial $(p<0,01)$, sendo que dentre o total de pessoas que tinham a doença, $80 \%(n=116)$ apresentaram incapacidade física no momento da avaliação (Tabela 3).

Tabela 3 - Distribuição de doenças crônicas não transmissíveis no pós-alta de hanseníase em relação à presença de Incapacidades físicas. Floriano e Picos,PI, Brasil, 2017 (continua)

\begin{tabular}{|c|c|c|c|c|c|c|}
\hline \multirow[t]{2}{*}{ Variáveis } & \multicolumn{2}{|c|}{ Sem incap. Física } & \multicolumn{2}{|c|}{ Com incap. física } & \multirow{2}{*}{$\frac{\text { Total }}{\mathbf{N}^{(1)}}$} & \multirow{2}{*}{ p valor } \\
\hline & $\mathbf{n}$ & $\%$ & $\mathbf{n}$ & $\%$ & & \\
\hline
\end{tabular}


Cogitare Enferm. (23)4: e58080, 2018

\begin{tabular}{|c|c|c|c|c|c|c|}
\hline Não & 158 & 30,86 & 354 & 69,14 & 512 & \multirow{2}{*}{0,15} \\
\hline Sim & 16 & 22,54 & 55 & 77,46 & 71 & \\
\hline \multicolumn{7}{|l|}{ Hipertensão $^{a}$} \\
\hline Não & 144 & 32,65 & 297 & 67,35 & 441 & \multirow{2}{*}{0,001} \\
\hline Sim & 29 & 20 & 116 & 80 & 145 & \\
\hline \multicolumn{7}{|c|}{ Outras comorbidades $^{b}$} \\
\hline AVC & 0 & 0 & 6 & 100 & 6 & \multirow{6}{*}{0,54} \\
\hline Artrose & 3 & 21,43 & 11 & 78,57 & 14 & \\
\hline Depressão & 1 & 20 & 4 & 80 & 5 & \\
\hline Hérnia de disco & 1 & 25 & 3 & 75 & 4 & \\
\hline Osteoporose & 1 & 10 & 9 & 90 & 0 & \\
\hline Outras & 28 & 30,43 & 64 & 69,57 & 92 & \\
\hline
\end{tabular}

${ }^{(a)}$ Teste Qui-quadrado de Pearson; ${ }^{(b)}$ Teste exato de Fisher; ${ }^{(1)}$ Os números totais (n) diferem em decorrência de valores missing.

\section{- DISCUSSÃO}

A presença de incapacidades e deformidades causadas pela hanseníase em um paciente pós-alta é indicador de que o diagnóstico foi tardio ou de que o seguimento clínico foi inadequado, o que amplia os desafios e a complexidade para a atenção pós-alta ${ }^{(9)}$. A ocorrência de GIF pode medir indiretamente a magnitude da hanseníase, pois a maioria dos pacientes não desenvolvem deformidades nos estágios iniciais da doença ${ }^{(10)}$. A prevalência de incapacidade física encontrada no estudo foi alta, superior aos resultados encontrados na literatura ${ }^{(7,11)}$. O número de casos com GIF 1 foi superior aos de GIF 2, consistente com dados oficiais e de estudos anteriores ${ }^{(1,8,12)}$.

Predominou o sexo feminino, semelhante a estudos realizados com pacientes atendidos em um programa de eliminação da hanseníase em São Luís-MA e em um Hospital Universitário do Ceará(12-13). Tem sido sugerido que a maior preocupação com a estética corporal e políticas específicas para a população feminina justificaria o predomínio desta entres os $\operatorname{casos}^{(12)}$. Com relação à idade, segundo o Ministério da Saúde, a hanseníase atinge principalmente a faixa etária economicamente ativa, que varia dos 20 aos $60 \operatorname{anos}^{(6)}$. A idade média obtida nos resultados da pesquisa foi maior do que a encontrada na literatura em estudos também com população no pós-alta ${ }^{(8,10-11)}$ evidenciando a característica de grande acometimento da população com idade mais avançada, entre os casos de hanseníase nos municípios da pesquisa.

O predomínio de pessoas com baixa ou nenhuma escolaridade reafirma que o baixo nível educacional é importante fator de risco à saúde. A baixa escolaridade é forte indicadora de condições precárias de moradia, nutrição, higiene e acesso aos serviços de saúde, fatores determinantes para a manutenção da cadeia de transmissão da hanseníase. Essa situação pode ampliar a vulnerabilidade dessas pessoas, podendo dificultar a compreensão, adoção de tratamento e de medidas para o autocuidado ${ }^{(8,14-16)}$.

A maior frequência de casos paucibacilares e da forma clínica indeterminada foi semelhante aos resultados referidos com predominância na literatura, ressaltando a importância do diagnóstico em tempo oportuno e adequado nos serviços de saúde, a partir de ações que visam o controle da hanseníase. Fatores importantes e que otimizam o diagnóstico são: o acesso adequado à assistência, a comunicação e educação em saúde ${ }^{(17)}$.

Aproximadamente 25 a 30\% dos pacientes com hanseníase desenvolvem reações hansênicas ou dano neural em algum momento. Dependendo da função do nervo no diagnóstico, quando é normal, $16 \%$ das pessoas podem ter reação, e, se o nervo estiver alterado, o índice pode atingir até $65 \%{ }^{(7)}$. O percentual de ocorrência de episódios reacionais diminui na medida em que aumenta o tempo após a conclusão da $\mathrm{PQT}^{(15)}$, o que pode explicar o percentual inferior a esses parâmetros encontrado no estudo, considerando que a população estudada já se encontrava no pós-alta por cura da hanseníase. Esse fato, porém, não exclui a importância de potencializar a atenção específica e multiprofissional a 
esse evento clínico, uma vez que a reação hansênica pode acarretar sérias complicações.

A estimativa da prevalência de incapacidade física foi maior nos homens quando comparados às mulheres, como foi visto em estudos, ${ }^{(11-12,15)}$ configurando, possivelmente, detecção mais tardia nesse público, haja vista que o acesso dos homens aos serviços da atenção primária à saúde na maioria dos casos limita-se apenas ao tratamento de uma morbidade ${ }^{(18)}$. O resultado da presente pesquisa foi semelhante a outro estudo(10) $q u e$, ao analisar a idade e o grau de incapacidade física, observou que há um aumento da idade em relação à presença de GIF, com média de idade de 34 anos para GIF 0 , 49 anos para GIF 1 e 57 anos para GIF 2. O diagnóstico realizado em pacientes com maior idade pode representar cronicidade da doença e possível instalação do dano neural.

Houve relação significativa entre a ocorrência de incapacidades e os menores níveis de instrução. É por meio da educação em saúde que as pessoas são sensibilizadas quanto aos primeiros sinais da hanseníase, a necessidade de diagnóstico precoce e prevenção de incapacidades. Diversos espaços comunitários podem ser utilizados para disseminar tal informação, como reuniões em grupo, sala de espera, propagandas ${ }^{(19)}$. Nesse sentido, a possível dificuldade na compreensão das orientações para tratamento, prevenção de incapacidades e medidas de autocuidado aumentam a chance de desenvolver ou agravar complicações ou deformidades.

A presença de algum grau de deficiência foi mais frequente em pessoas que tiveram as formas clínicas Virchowiana e Boderline em relação àquelas que tiveram formas paucibacilares (indeterminada e tuberculoide). Os pacientes multibacilares aumentaram 5,7 vezes as chances de apresentar incapacidades quando comparados aos paucibacilares. Esse resultado pode estar relacionado ao fato de que os casos multibacilares são diagnosticados mais tardiamente, o que pode propiciar a instalação de incapacidades intimamente ligadas ao tempo ${ }^{(11,20)}$.

Foi encontrada grande quantidade de pessoas que referiu não ter recebido a avaliação neurológica simplificada. Esse fato não está de acordo com o que é preconizado pelo Ministério da Saúde ${ }^{(6)}$ e prejudica inteiramente o seguimento dos casos e a qualidade da assistência à pessoa com hanseníase.

No tocante à sobreposição de doenças, o estudo foi semelhante aos resultados de uma pesquisa realizada em São José do Rio Preto-SP no ano de 2012, no qual as doenças frequentemente associadas foram diabetes e hipertensão arterial ${ }^{(8)}$. Neste mesmo estudo, não foi encontrada diferença estatística em relação à presença de incapacidades físicas e àqueles que apresentavam alguma doença associada. A partir dos resultados encontrados na presente pesquisa, compreendeu-se que o fato de apresentar alguma comorbidade não é um fator de risco para o desenvolvimento de incapacidades físicas. No entanto, comprovou-se que, na presença de incapacidade física, a frequência de diabéticos e hipertensos foi maior quando comparado ao grupo que não apresentou incapacidade.

A lenta instalação do processo infeccioso associado à hanseníase, a baixa letalidade e a ocorrência de eventos imunológicos relacionados compõem um quadro que favorece a amplificação da morbimortalidade nas populações acometidas quando da associação com outras doenças. Questões como isolamento, estigma, restrição à participação social, limitação funcional e incapacidade física não apenas ampliam os desafios para o manejo da hanseníase como condição crônica, mas também aumentam a frequência e a magnitude de fatores de risco para outras doenças crônicas não transmissíveis (DCNT) ${ }^{(17)}$.

Deve ser considerado ainda o grande número de pessoas acometidas por essas doenças atualmente, principalmente as pessoas idosas, faixa etária predominante na pesquisa. Segundo dados Pesquisa Nacional de Saúde, realizada pelo Ministério da Saúde em parceria com o Instituto Brasileiro de Geografia e Estatística (IBGE), cerca de $40 \%$ da população adulta brasileira, o equivalente a 57,4 milhões de pessoas, possui pelo menos uma DCNT, dentre estas se destacando a hipertensão arterial e o diabetes, que atingem $21,4 \%$ e $6,2 \%$ da população, respectivamente.

No Brasil, ainda é verificado o diagnóstico tardio da hanseníase, mesmo após os primeiros sintomas. O Ministério da Saúde e outras organizações realizam projetos e campanhas destacando a busca pela identificação precoce dos sinais e sintomas da doença, além de buscar a promoção de condutas adequadas e eficazes para o controle da doença e qualidade devida ${ }^{(7)}$.

Como limitação do estudo, destaca-se a qualidade do banco SINAN em relação à completitude e 
inconsistência das informações que gerou dificuldades quanto à localização dos casos, problemática reparada através do cruzamento com outros bancos CADSUS, SIM que foram disponibilizados. Consideram-se relevantes estudos que envolvam a utilização desse sistema, pois permitirão avaliações congruentes à situação epidemiológica dos agravos de saúde.

\section{CONCLUSÃO}

A sobreposição de doenças não se comportou como um fator de risco para a ocorrência de incapacidades físicas, mas constatou-se frequência importante de pessoas com doenças associadas acometidas com algum grau de incapacidade.

O estudo evidenciou dados preocupantes acerca da situação das pessoas no pós-alta da hanseníase nos municípios avaliados, levando a reflexão sobre a qualidade das ações e serviços dispensados pelas unidades de saúde referentes à atenção à pessoa com hanseníase. A partir do conhecimento da situação do pós-alta das pessoas acometidas pela hanseníase, é possível realizar planejamento de ações de saúde mais adequadas à população, por meio da identificação precoce de situações de vulnerabilidade.

Recomendam-se capacitações para os profissionais da atenção primária (médicos, enfermeiros, odontólogos e ACS) e também para os profissionais dos serviços de nível secundário e terciário que poderão receber esses indivíduos no pós-alta, na perspectiva de prevenção e tratamento de incapacidades físicas decorrentes da hanseníase. Importante também a construção de uma linha de cuidado, com foco na rede de atenção, que fortaleça a longitudinalidade do cuidado.

\section{REFERÊNCIAS}

1. Neves TV, Valentim IM, Vasconcelos KB, Rocha ESD, Nobre MSRS, Castro JGD. Perfil de pacientes com incapacidades físicas por hanseníase tratados na cidade de Palmas- Tocantins. Revista Eletrônica Gestão\& Saúde. [Internet].2013 [acesso em 2017 nov 02];4(2). Disponível em: http://dx.doi.org/10.18673/gs.v4i2.22936.

2. Rosa GR, Lima MM,Brito WI , Moreira AM. Análise da completude de incapacidade em hanseníase da regional de saúde de Rondonópolis/MT. Revista Eletrônica Gestão \& Saúde.[Internet]. 2016[acesso em 2017 nov 29];7(1). Disponível em: http://dx.doi.org/10.18673/gs.v7i1.22068

3. World Health Organization (WHO). Weekly epidemiological record [Internet]. Geneva: World Health Organization; 2017 [acesso em 2018 ago 12]. Disponível:http://apps.who.int/iris/bitstream/handle/10665/258841/ WER9235.pdf;jsessionid=7745CE443F3C12739E5698B3820B3C73? sequence $=1$.

4.Araújo TME, Felinto DA, Neto SAS, Macêdo SF, Silva GV, Sousa ALG. Boletim de vigilância em saúde do estado do Piauí: Hanseníase 2014. Teresina: EDUFPI,2016.

5.Alencar MJF. Reações hansênicas em pós-alta de Poliquimioterapia: fatores associados e visão dos usuários numa área endêmica do Brasil [tese]. Fortaleza (CE): Universidade Federal do Ceará;2012.

6.Ministério da Saúde (BR). Departamento de Vigilância Epidemiológica, Secretaria de Vigilância à Saúde, Ministério da Saúde. Guia prático sobre a hanseníase. Brasília: Ministério da Saúde;2017. 70 p.

7.Barbosa JC, Ramos NA Jr, Alencar OM, Pinto MSP, Castro CGJ. Atenção pós-alta em hanseníase no Sistema Único de Saúde: aspectos relativos ao acesso na região Nordeste. Cad. Saude Colet.[Internet].2014[acesso em 2017 nov 30];22(4). Disponível em:http://dx.doi.org/10.1590/1414-462X201400040008.

8. Monteiro LD, Alencar CHM, Barbosa JC, Braga KP, Castro MD, HeukelbachJ. Incapacidades físicas em pessoas acometidas pela hanseníase no período pós-alta da poliquimioterapia em um município no Norte do Brasil. Cad Saúde Pública. [Internet].2013 [acesso em 2017 dez 05];29(5). Disponível em: http://dx.doi.org/10.1590/S0102$311 X 2013000500009$.

9.Rodrigues LC, LockwoodDNJ. Leprosynow: epidemiology, progress, challenges, andresearch gaps. Lancet InfectDis.[Internet].2011 [acesso em 2017 dez 05];11(6). Disponível em:https://doi.org/10.1016/S14733099(11)70006-8. 
10.Souza GL. Incapacidade física em pessoas afetadas pela Hanseníase: pós-alta [tese]. Belém (PA): Universidade Federal do Pará;2013.

11.Nardi SMT, Paschoal VD, Chiaravalloti-Neto F, Zanetta DMT. Deficiências após a alta medicamentosa da hanseníase: prevalência e distribuição espacial. Rev Saúde Pública. [Internet].2012[acesso em 2017 dez 05];46(6). Disponível em:http://dx.doi.org/10.1590/S0034-89102013005000002.

12.Araújo AERA, Aquino DMC, Goulart IMB, Pereira SRF, Figueiredo IA, Serra HO et al. Complicações neurais e incapacidades em hanseníase em capital do nordeste brasileiro com alta endemicidade. Rev BrasEpidemiol. [Internet].2014[acesso em 2017 dez 07];17(4). Disponível em: http://dx.doi.org/10.1590/1809-4503201400040009

13.Queirós MI, Ramos NA Jr, Alencar CHM, Monteiro LD, Sena AL, Barbosa JC. Perfil clínicoepidemiológico de pacientes com hanseníase atendidos em hospital universitário no Ceará entre 2007 e 2011.AnBrasDermatol. [Internet].2016 [acesso em 2017 dez 07];91(3). Disponível em:http://www.anaisdedermatologia.org.br/ detalhe-artigo/102471/Perfil-clinico-epidemiologico-de-pacientes-com-hanseniase-atendidos-em-hospitaluniversitario-no-Ceara-entre-2007-e-2011-

14.Buna ATM, Rocha FCG, Alves EM, Granja FBC, Sousa DJ, Silva MGP. Incapacidades físicas nos pacientes com hanseníase cadastrados em uma unidade de saúde de São Luís - MA. RevInterd. [Internet].2015[acesso em 2017 dez 07]; 8(1) Disponível em:https://revistainterdisciplinar.uninovafapi.edu.br/index.php/revinter/article/ view/579/pdf_189.

15.Ribeiro GC, Lana FCF. Incapacidades físicas em hanseníase: caracterização, fatores relacionados e evolução. Cogitare enferm. [Internet].2015 [acesso em 2017 dez 12],20(3). Disponível em:http://dx.doi.org/10.5380/ ce.v20i3.41246.

16. Miranzi SSC, Pereira LHM, Nunes AA. Perfil epidemiológico da hanseníase em um município brasileiro, no período de 2000 a 2006. Rev SocBrasMed Trop.[Internet].2010 [acesso em 2017 dez 12];43(1). Disponível em: http://www.epi.uff.br/wp-content/uploads/2016/09/hans.pdf.

17.Cunha MD, Cavaliere FA, Hercules FM, Duraes SM, Oliveira ML, Matos HJ. Os indicadores da hanseníase e as estratégias de eliminação da doença, em município endêmico do Estado do Rio de Janeiro, Brasil. Cad Saúde Publica [Internet].2007[acesso em 2017 dez 12];23(5). Disponível em: http://dx.doi.org/10.1590/S0102311X2007000500020.

18.Silva DM, Souza TO, Lima MV, Yarid SD, Sena ELS. Acessibilidade do homem aos serviços da atenção básica: uma aproximação com a bioética da proteção. Cogitareenferm. [Internet].2013 [acesso em 2017 dez 18];18(3). Disponível em: http://dx.doi.org/10.5380/ce.v18i3.46361.

19. Moreira AJ, Naves JM, Fernandes LFRM, Castro SS, Walsh IAP. Ação educativa sobre hanseníase na população usuária das unidades básicas de saúde de Uberaba-MG. Saúde debate.[Internet].2014 [acesso em 2017 dez 18];38(101). Disponível em:http://dx.doi.org/10.5935/0103-1104.20140021.

20.Moschioni C, Antunes CMF, Grossi MAF, Lambertucci JR. Riskfactors for physicaldisabilityatdiagnosisof 19,283 new cases ofleprosy. RevSocBrasMed Trop. [Internet].2010 [acesso em 2017 dez 18];43(1). Disponível em: http:// dx.doi.org/10.1590/S0037-86822010000100005. 